\title{
Cost-effective ROADM Design to Maximize the Traffic Load Capacity of u-DWDM Coherent Metro- Access Networks
}

\author{
Samael Sarmiento, Salvatore Spadaro, and Jose A. Lazaro \\ Advanced Broadband Communications Center (CCABA), Universitat Politècnica de Catalunya (UPC), Jordi \\ Girona 1-3, 08034 Barcelona Spain, e-mail: samael.sarmiento@tsc.upc.edu
}

\begin{abstract}
The current constant growing in traffic demands caused by the popularization of cloud services, mobile and social networks is requiring architectural changes at the underlying networks so as to provide a more highly dynamic connectivity. Cost-effective and energy efficient solutions are also required in flexible network subsystems in order to make available future sustainable networks. In this context, a novel cost-effective and energy-efficient solution DWDM ROADM (D-ROADM) node design has been recently considered to enable optical Metro-Access networks convergence. In this paper, we assess the D-ROADM capabilities in a dynamic ultra-Dense Wavelength Multiplexing (uDWDM) coherent Ring Network scenario. In particular, the metric Blocking Bandwidth Probability (BBP) vs. Traffic Load Capacity (TLC) has been considered. Our numerical evaluations show that the performance penalty of cost-effective D-ROADM based networks could be reduced to $35 \%$ for $62.5 \mathrm{GHz}$ DWDM channels when compared to WSS-based ROADMs.
\end{abstract}

\section{INTRODUCTION}

The telecommunication scenario has been rapidly and dramatically changing in the last years. The emergence of new cloud and multimedia streaming services such as online video on PCs and smartphones, online gaming, and high-definition television over the Internet, along with the deployment of the Internet of Things (IOT) and the convergence between optical and wireless communications at the $5 \mathrm{G}$ paradigm [1] are requiring changes to the networks in order to enable scalable growth in traffic volume, while supporting a high level of dynamic connectivity, full flexibility and increased energy-efficiency. As a consequence of that, Metro Network is converging with Access Networks and evolving towards the adoption of all-optical solutions [2,3]. In this context, transmission techniques based on ultra-Dense Wavelength Multiplexing (u-DWDM) are a promising alternative to Time Division Multiplexing (TDM) solutions due to their high spectral efficiency [4], which is accomplished by the subdivision of a DWDM channel into smaller channels, called frequency slots (FSs), wherein the up-link (U) and the down-link (D) for each user can be established (see Fig. 1) [5, 6]. 


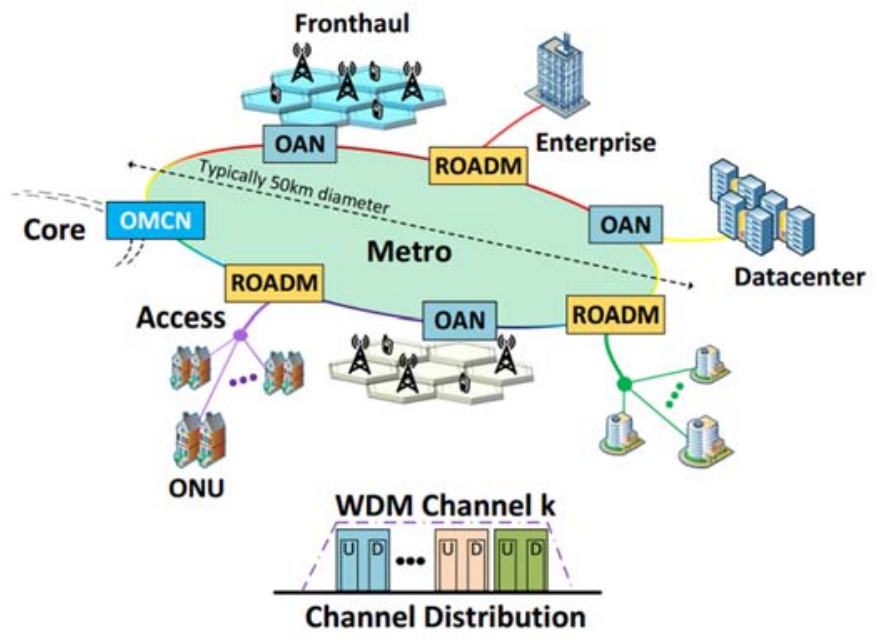

Fig. 1. Flexible $5 G$ Metro-Access Network scenario. Inlet: considered flexible ultra-dense WDM frequency slot division.

According to the scenario shown in Fig. 1, the Metro-Access Network relies on different node elements, that is the Optical Metro-Core Node (OMCN), the Optical Aggregation Node (OAN), the Optical Network Unit (ONU) and the reconfigurable optical add-drop multiplexer (ROADM); all of them are detailed in [7]. In particular, ROADMs are all-optical nodes that connect the Metro to the Access Networks while the OMCN, OAN and ONU still perform Optical-Electrical-Optical (OEO) conversion for their functions and they are the nodes that allow the transfer of information between the Metro and Core Network, the Metro Network and a Datacenter, or a 5G-Fronthaul and the access network and users, respectively. In general, ROADMs are considered as the key element for future Metro-Access converged networks because they reduce the instances of OEO conversions by setting all-optical links between metro and access nodes. Benefits of this solution include terabits per second bandwidth with low bit error rates, lower network's power consumption, data transparency, and freedom from interference leading to a secure communication system.

The ROADM design is basically driven by new network-level requirements, such as full flexibility, adaptability, scalability, resilience and increased energy-efficiency [8]. In order to reach those features, two alternatives colorless directionless (CD) ROADM architectures, which can be used in the scenario shown in Fig. 1, have been proposed and analyzed in [9], including a detailed cost comparison between them. The first ROADM solution, fully flexible and compacted but far more expensive [9], is based on wavelength selective switches (WSS) that can work with FS granularity. Using this WSS-based ROADM, the add/drop (A/D) and passthrough (PT) functions can be run over each FS individually (see Fig. 2). The second ROADM solution, more cost-effective and modular [9], works with DWDM channel granularity. In particular, a module consists of two DWDM fixed filters and two switches that manage one DWDM channel (see Fig. 2). Then, the A/D and PT functions can be applied on a group of FSs at the DWDM channels of the ROADM (D-ROADM), see Fig. 2. For a group of FSs whose DWDM channel is not supported by the D-ROADM, it is transparent for them, achieving vendors' requirements. Taking into account that the D-ROADM switches a group of FSs, the main target of the paper is to demonstrate if networks based on cost-effective D-ROADM with a low 
number of modules $(N)$ could achieve a similar Traffic Load Capacity $(T L C)$ as networks using colorless WSS-based ROADMs.
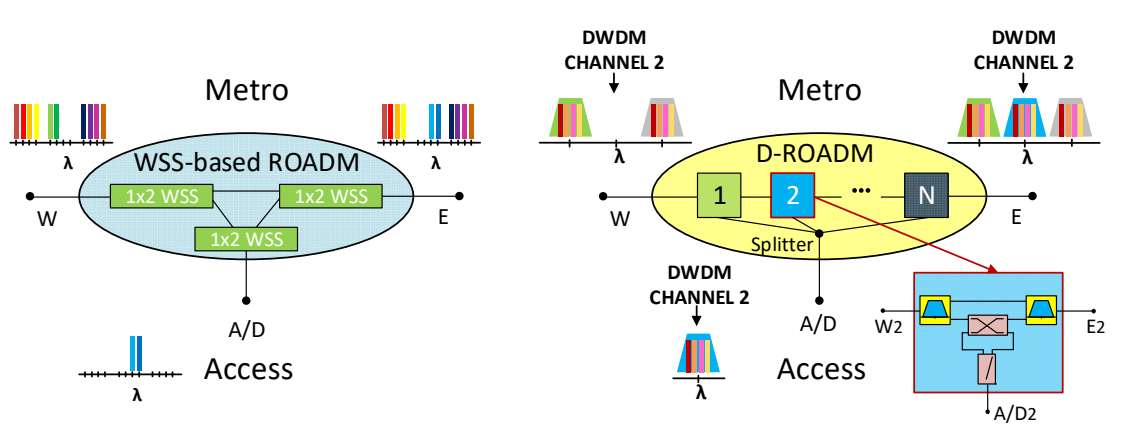

Fig. 2. WSS-based ROADM vs. DWDM ROADM (D-ROADM) functionalities.

The remainder of this paper is structured as follows: Section II presents the problem statement for the design of Metro-Access networks based on D-ROADMs, two different heuristics for the design of the D-ROADMs of a network as well as the details and assumptions of the evaluated u-DWDM coherent Metro-Access networks scenario; Section III shows the obtained numerical results of evaluating both heuristics with regarding to the Traffic Load Capacity (TLC) of the networks against to the same networks using WSS-based ROADMs; finally, Section IV completes the paper with the main conclusions.

\section{OPTICAL METRO-ACCESS NETWORKS DESIGN BASED ON COST-EFFECTIVE D- ROADMS}

In this section, we first present the design of metro-access converged networks based on DROADMs knowing that each module of the D-ROADM switches a group of FSs and the ROADM's optical insertion losses increase with the number of modules $(N)$, as it is indicated in [9]. Keeping that in mind, two different heuristics to find out the best D-ROADMs' design of a network that optimize its TLC value have been analyzed. Eventually, we introduce the scenario details as well as the assumptions considered in our study.

\section{A. Problem statement}

In our study, three important aspects must be considered:

1. The first one is that the D-ROADM switches all frequency slots of a DWDM channel in the same direction through the settled configuration of the switches of the module associated with each DWDM channel (i.e. from/to A/D port to/from E port in Fig. 2), blocking of any connection, for this specific DWDM channel, in the other two possible directions of the ROADM's module (i.e. from/to E port to/from $W$ port and from/to $A / D$ port to/from $W$ port when the A/D mode is selected to work over E port, see Fig. 2). It is because of the DROADM that does not support the contentionless (C) feature [9]. It can cause that the resulting logical graph from the union of each DWDM channel's equivalent graph of the network is disconnected if the D-ROADM design is not properly managed. In this way, a bad design of the network D-ROADMs can prevent the establishment of connections between every possible pair of source-destination nodes of the network since may not be feasible to find a path for any DWDM channel of the network. Figure 3(a) presents an 
example of the design of the D-ROADMs for a three-DWDM-channel network that permits to highlight the mentioned problem. For each DWDM channel of the network shown in the example [see Fig. 3(a)], all possible logical paths are schematically depicted, as well as the channels assigned to each D-ROADM. The design strategy chosen for this example consists of assigning all channels to each D-ROADM and configuring the switches of each ROADM's module to work in add/drop mode over its different ring ports (W port or E port). That is, a module settled allowing paths between A/D port and W port, and the others two modules for $A / D$ port and $E$ port. That strategy can in principle seem appropriate as the traffic demands of both PONs can be routed by two different physical paths. Nevertheless, making a deep inspection of the network, we can realize that the resulting network's graph is disconnected. In this way, for example, there is not possible a path between nodes with IDs 1 and 4, and nodes with IDs 2 and 4 . In order to resolve that problem two different heuristics will be presented in Sections II.B and II.C.

2. The second aspect to consider is that the D-ROADM provides higher switching flexibility when a higher number of modules is used. However, the D-ROADM insertion loss increases with the number of modules $(N)$ due to the use of a power splitter (see Fig. 2) to combine the DWDM channels supported by the D-ROADM [9].

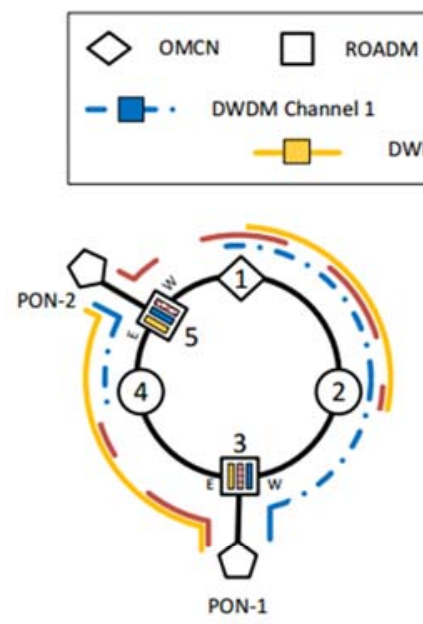

(a)

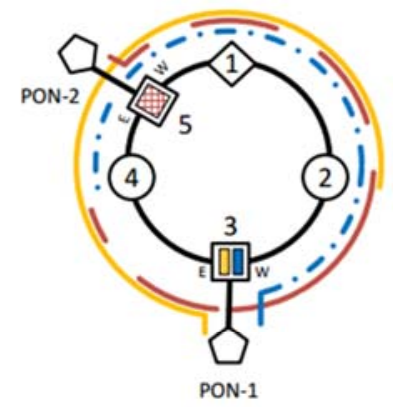

(b)

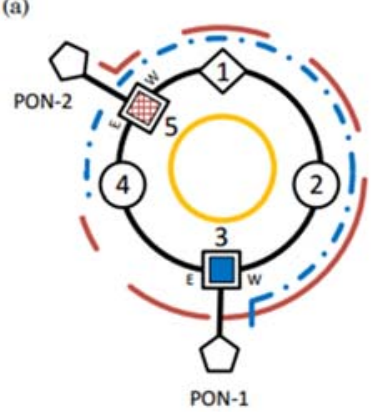

(c)

Fig. 3. Three different examples of design of the D-ROADMs of a simple ring-based network whose resulting logical graphs from the union of the equivalent graph for each DWDM channel of the network are: (a) disconnected and $(b, c)$ not disconnected.

3. Finally, the third aspect is that it is considered a dynamic scenario in which demands arrive at and depart from the network in a random manner. Moreover, it is also considered that 
traffic demands are distributed between all pair of source-destination nodes of the network randomly. Then, depending on the profile of the traffic demands to be satisfied, the D-ROADM's design, that is, its number of modules should be adapted to reduce the total traffic demands blocked.

Taking into account the three aspects previously discussed, the problem to resolve here is to find out the minimum number of modules of each D-ROADM of the network and their switches configuration that best suit to the traffic profile loaded to the network, while keeping the D-ROADM insertion losses as low as possible. In this way, the efficient design of the DROADM may lose the colorless feature, but its implementation cost is significantly reduced as detailed in [9] and keeps adapted to traffic demands.

A greedy approach to determine the set-up of each D-ROADM that best suits to the traffic profile arrived at the network would be to consider firstly that each D-ROADM is able to commute all the DWDM channels of the network. In this way, the problem is simplified to search for the best set-up of the D-ROADM module's switches. Then, the useless modules or the modules which are configured to work as pass-through mode have to be removed. However, this technique dramatically increases the computational complexity with the number of ROADMs ( $\mathrm{N}_{\text {ROADMs }}$ ) and channels ( $\mathrm{N}_{\text {channels }}$ ) of the network. Specifically, knowing that each module of the D-ROADM has three possible operation modes [9], $3^{\mathrm{N}_{\text {channels }}} *_{\text {ROADMs }}$ different network configurations have to be analysed to learn the network configuration that best suits to the traffic profile which arrives at the network. This means, for each obtained configuration, the dynamic Routing and Spectrum Assignment (RSA) problem for all traffic demands [10], has to be solved. Thus, for a simple network as shown in Fig. 3 in which $\mathrm{N}_{\text {ROADMs }}=2$ and $\mathrm{N}_{\text {channels }}=3$, the number of times that the dynamic RSA must be resolved for all traffic demands is 729 times, while for the same network working in the all $C$ band, i.e. $\mathrm{N}_{\text {channels }}=20$ using modules with $125 \mathrm{GHz}$ of effective bandwidth [9], is $\sim 1.2 \cdot 10^{19}$, doing this technique strongly unviable. Alternatively, the following heuristics can be used for obtaining a design of the ROADMs of a network that best suit to the traffic profile. Using these techniques, the maximum number of times that the dynamic RSA problem must be solved for all traffic demands arrived at the network is lower than $\mathrm{N}_{\text {channels }}$ of the network as it shall be shown in Sections 2.B and 2.C. To ease the comprehension of the proposed heuristics, Table 1 details the notations used in their description. 


\begin{tabular}{|c|c|}
\hline Symbol & Definition \\
\hline $\mathcal{G}=(\mathcal{N}, \mathcal{E})$ & $\begin{array}{l}\text { Graph representing the optical network, where } \mathcal{N} \text { is the set of nodes } \\
\text { (OMCN, OAN, ROADM and ONU) and } \mathcal{E} \text { is the set of optical links of the } \\
\text { analyzed network. }\end{array}$ \\
\hline $\mathrm{BW}_{\mathrm{N}}$ & Total Network Bandwidth \\
\hline $\mathrm{BW}_{\mathrm{DWDM}-\mathrm{C}}$ & DWDM Channel Bandwidth \\
\hline $\mathrm{PB}_{\mathrm{o}}$ & Power Budget available per connection \\
\hline $\mathrm{P}_{\mathrm{WC}}$ & $\begin{array}{l}\text { Worst Case Path, defined as the path between a pair of source-destination } \\
\text { nodes which presents the highest total attenuation of the network. }\end{array}$ \\
\hline $\mathcal{D}$ & $\begin{array}{l}\text { Dynamic traffic demands profile loaded to the network. This traffic profile } \\
\text { is characterized by: (i) how the traffic is distributed between the nodes of } \\
\text { the network (e.g. uniformly), (ii) what traffic arrival process follows and } \\
\text { what type of distribution presents the durations (e.g. Poisson and negative } \\
\text { exponential, respectively) and (iii), the average requested bitrate per } \\
\text { demand, } \bar{C} \text {, arrived at the network. }\end{array}$ \\
\hline$\rho$ & Traffic load (in erlang units) \\
\hline TLC & Traffic load capacity, defined as $\rho * \overline{\mathrm{C}}$ (bps). \\
\hline $\mathrm{BBP}$ & $\begin{array}{l}\text { Blocking Bandwidth Probability, defined as the volume of rejected traffic } \\
\text { divided by the volume of all traffic offered to the network. }\end{array}$ \\
\hline$B D_{\text {ring }}$ & $\begin{array}{l}\text { Number of blocked demands which have their origin and destination in a } \\
\text { node of the ring part of the network. }\end{array}$ \\
\hline$B D_{P O N-i}$ & $\begin{array}{l}\text { Number of blocked demands which have their origin or destination in a } \\
\text { node belonging to PON-i. }\end{array}$ \\
\hline$R S A(\mathcal{G}, \mathcal{D}, B B P)$ & $\begin{array}{l}\text { Sub-routine resolves the dynamic Routing and Spectrum Assignment of all } \\
\text { traffic demands arrived at the network in the simulations. The input } \\
\text { parameters are the network model }(\mathcal{G}) \text {, the traffic demands profile loaded } \\
\text { to the network }(\mathcal{D}) \text { and the considered Blocking Bandwidth Probability } \\
(\mathrm{BBP}) \text {. The output parameters are the TLC of } \mathcal{G} \text { for the considered BBP } \\
\text { value and the number of blocked demands of the different network's parts } \\
\left(B D_{\text {ring }} \text { and } B D_{P O N-i}\right) \text {. Pre-computation of the two-lowest loss path } \\
\text { between all pair of source-destination of the network and the first-fit } \\
\text { policy for allocating FSs are considered to resolve the dynamic RSA. }\end{array}$ \\
\hline
\end{tabular}

Table 1. Notations used in the description of the D-ROADM designs heuristics.

\section{B. Heuristic I}

A strategy that allows resolving the mentioned problem of the logical disconnection of the network is shown in Fig. 3(b). This strategy is based on assigning a different channel or channels between the D-ROADMs of the network and settling the switches of each module associate with each channel to work in add/drop mode over any ring port of the D-ROADM (E port or W port) as it is shown in Fig. 3(b).

As it can be seen, the connection between all possible nodes of the network can be established using the available resources. In this strategy, it is considered that for allocating traffic demands between nodes belonging to the ring part of the network and between nodes of the ring and nodes of a PON, it must be used the same available resources. Thus, for example, for allocating traffic demands between node with ID 4 and nodes of the PON-2, the DWDM Channel 2 can be used for such purpose [Fig. 3(b)]. On the contrary, if a traffic demand presents the origin and destination in a node belonging to the ring part of the network, the available frequency slots of any possible channel can be used. For example, for establishing a connection between nodes with IDs 1 and 4 shown in Fig. 3(b), the available set of FSs of the three DWDM channels of the considered network can be utilized. At the same time, distributing the available DWDM channels between all the D-ROADMs of the network reduces the number of modules $(N)$ per D-ROADM and consequently the D-ROADM insertion losses. 
Figure 4 shows a pseudo-code for the proposed heuristic. The input parameters are the model of the network $(\mathcal{G})$, the network bandwidth $\left(\mathrm{BW}_{\mathrm{N}}\right)$ that is shared by all users, the considered DWDM channel bandwidth $\left(\mathrm{BW}_{\mathrm{DWDM}-\mathrm{C}}\right)$ which coincides with the bandwidth of each $\mathrm{D}$ ROADM module, the considered power budget value $\left(P B_{o}\right)$ per connection, depending on the chosen modulation format technology, to the received signal can rightly be detected, the traffic nature loaded to the network, defined by $\mathcal{D}$, and the objective $B B P$ value. The output is the network design that maximizes the $T L C$ of the network using the strategy introduced in Fig. 3(b). As we can deduce from Fig. 4, to make a good management of the network, its number of DWDM channels $\left(M N C_{N}=\mathrm{BW}_{\mathrm{N}} / \mathrm{BW}_{\mathrm{DWDM}-\mathrm{C}}\right)$ must be greater than or equal to the number of D-ROADMs ( $N_{R O A D M S}$ ) of the network. On the other hand, new modules can be added to the D-ROADMs as required according to $\mathcal{D}$ so as to improve the network's TLC for a given $B B P$ as long as the worst path attenuation case $\left(\mathrm{P}_{\mathrm{WC}}\right)$ of the network is lower than the considered power budget per connection $\left(\mathrm{PB}_{\mathrm{o}}\right)$. Then, once an initial network design is obtained (NetworkSolutionDesign ${ }_{0}$ ), the dynamic RSA for all traffic demands is resolved to analyze the origin and destination of blocked demands. Extra DWDM channels are, thus, added to the D-ROADMs to allocate those demands. This is recursively done till exhausting the free DWDMs channels. Therefore, this heuristic implements an efficient distribution of the available DWDM channels among the D-ROADMs of the network. From Fig. 4, the number of times that the dynamic RSA problem has to be resolved for all traffic demands arrived at the network to obtain the most efficient network design is $2 *\left(M N C_{N}-M N C_{N_{0}}\right)+1$, where $M N C_{N}$ is the number of DWDM channels of the network, previously defined, and $M N C_{N_{0}}$ is the required number of DWDM channels to the first network design.

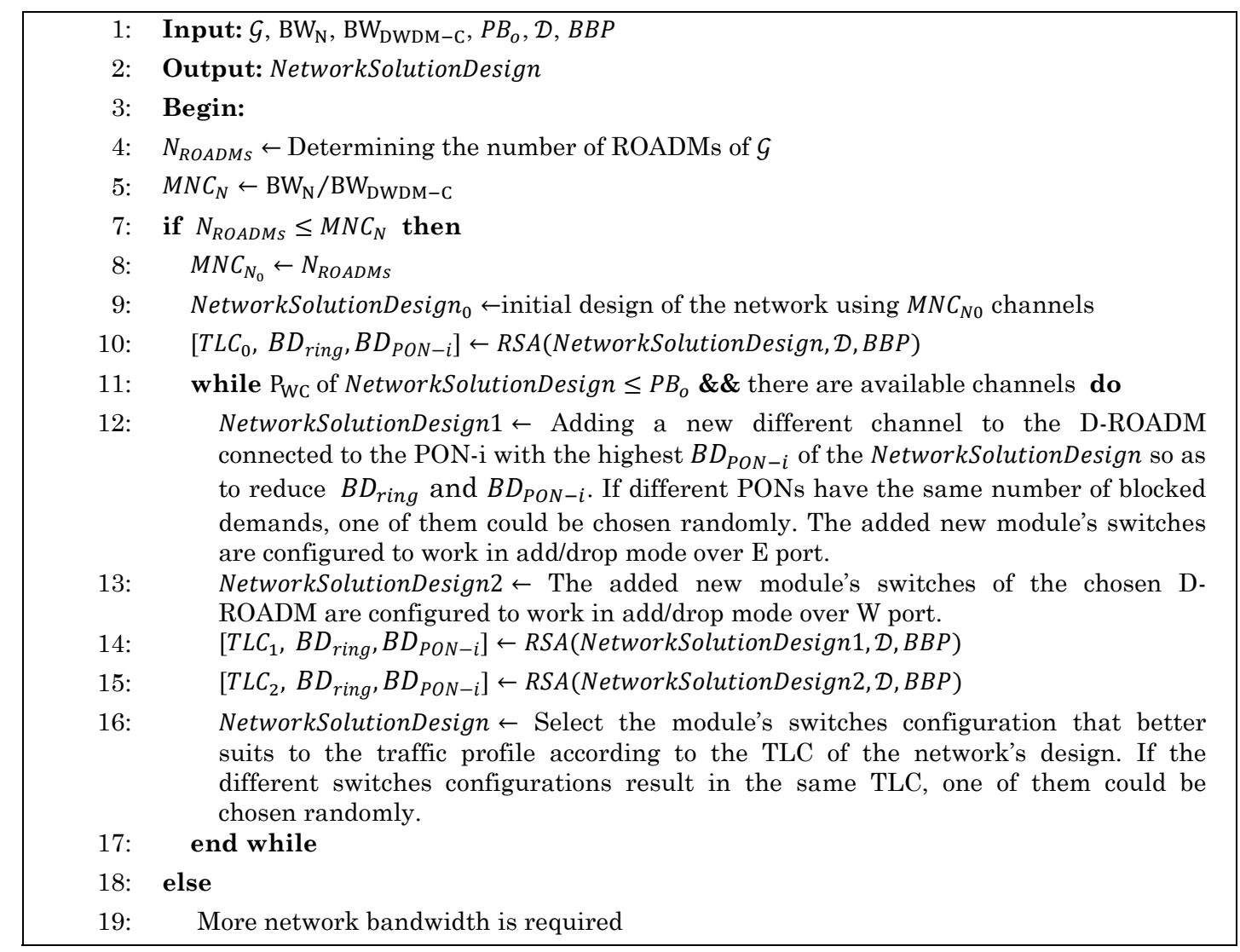


$\begin{array}{ll}\text { 20: } & \text { end if } \\ \text { 21: } & \text { return NetworkSolutionDesign }\end{array}$

Fig. 4. Pseudo-code of the proposed heuristic I.

\section{Heuristic II}

Another strategy that also provides a full logical connection among all the network nodes is introduced in Fig. 3(c). That strategy consists of booking one of the available channels for the connections between nodes pertaining to the ring part of the network and using the others two channels for the traffic of the PONs. That is, the DWDM channel 3 can just be used for allocating traffic demands between nodes 1, 2 and 4, and the channels 1 and 2 for traffic demands of the PONs. This strategy is more efficient that the heuristic previously presented as it shall be proved in the next section although it requires a smarter allocation of the DWDM channels as the network size increases.

Figure 5 shows a pseudo-code of the proposed heuristic II. The input parameters are the same than the heuristic explained in the previous sub-section. The output is the best network design that maximize the TLC of the network. To illustrate the proposed heuristic II, it will be applied over the Metro-Access Network example shown in Fig. 7. This example, which will be one of the studied network examples in the next section, considers that the network bandwidth $\left(\mathrm{BW}_{\mathrm{N}}\right)$ is of $750 \mathrm{GHz}$ and the DWDM channel bandwidth $\left(\mathrm{BW}_{\mathrm{DWDM}-\mathrm{C}}\right)$ is $125 \mathrm{GHz}$ [9]. The proposed heuristic can be divided into three smaller sub-problems.

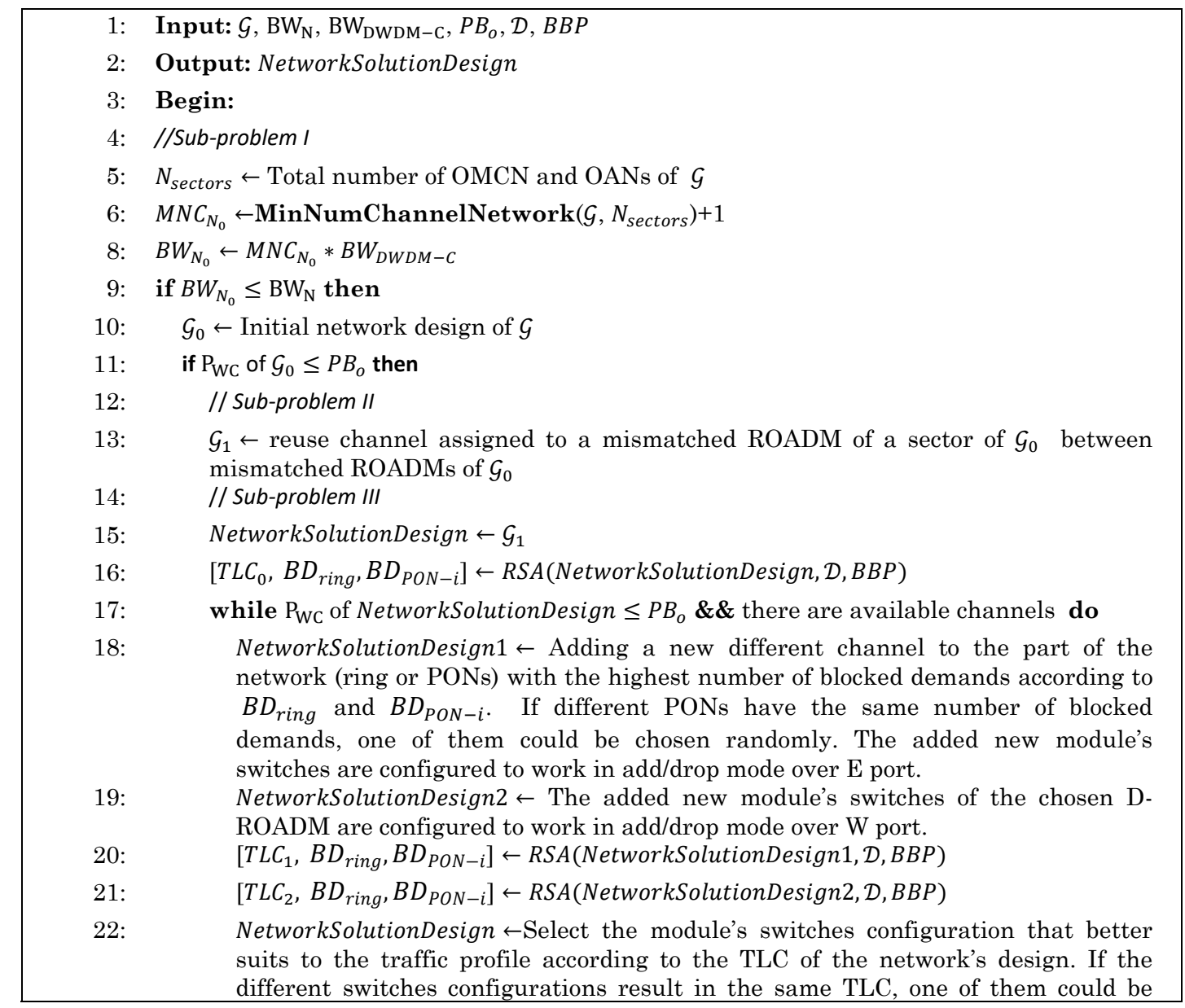




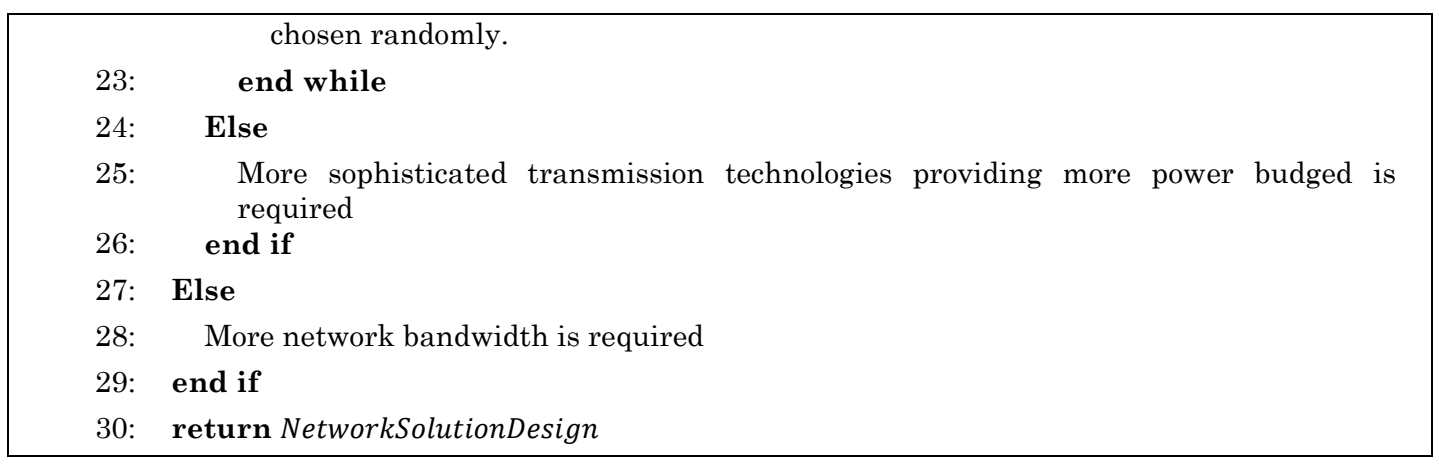

Fig. 5. Pseudo-code of the proposed heuristic II.

The first sub-problem involves finding out the minimum number of channels $\left(\mathrm{MNC}_{\mathrm{N}_{0}}\right)$ that are required in order to make possible to establish the connection between every pair of sourcedestination nodes of the network. In other words, it must be determined the minimum number of DWDM channels which are required to avoid that the resulting graph of the network is disconnected following the strategy shown in Fig. 3(c). In that strategy, traffic demands between nodes of the ring part of the network and between ring nodes and nodes of a PON use different DWDM channels. Figure 6 shows the proposed heuristic that can be used in order to reach that goal (MinNumChannelNetwork, in line 6 of the pseudo-code shown in Fig. 5). This heuristic basically consists on dividing the ring part of the network in a certain number of sectors $\left(\mathrm{N}_{\text {sectors }}\right)$ and determining the minimum number of DWDM channels that are required in each sector in order to ensure that every possible connection between nodes of a PON connected to a D-ROADM of the sector and a node of the ring part of the network can be established. In particular, a sector is defined as a pair of two consecutives nodes with opto-electrical conversion functionalities (OMCN or OANs) among which there can or cannot be D-ROADMs. Figure 7(a) shows the way of how the considered network can be divided into different sectors. In particular, the network is divided into three sectors (sector I, II and III) because the network has one OMCN and two OANs. For determining the number of different channels that are required for the D-ROADMs in each sector, the following rule considered in the pseudo-code shown in Fig. 6 can be used:

- If the number of ROADMs of the sector $\left(N_{R O A D M S}\right)$ is even, the number of required channels is $N_{R O A D M S} / 2$. It is due to the fact that a DWDM channel can be assigned to two different ROADMs of the same sector but with opposite switches' configuration as it can be noticed in Fig. 7(a), sector I, in which the DWDM channel 2 has been assigned to ROADMs with IDs 7 and 8.

- If $N_{R O A D M S}$ is odd, an extra channel is required for the ROADM that is mismatched. For example, the DWDM channel 1 has been committed to the ROADM with ID 9 of the sector $I$ which is exhibited in Fig. 7(a).

Once determined the number of channels for the D-ROADMs of each sector of the network, and according to the strategy shown in Fig. 3(c), a new channel must be added for allocating the traffic demands between nodes of the ring part of the network. So the minimal number of required channels in the considered network example (see Fig. 7) is five of the available six channels.

1: $\quad$ Input: $\mathcal{G}, N_{\text {sectors }}$ 


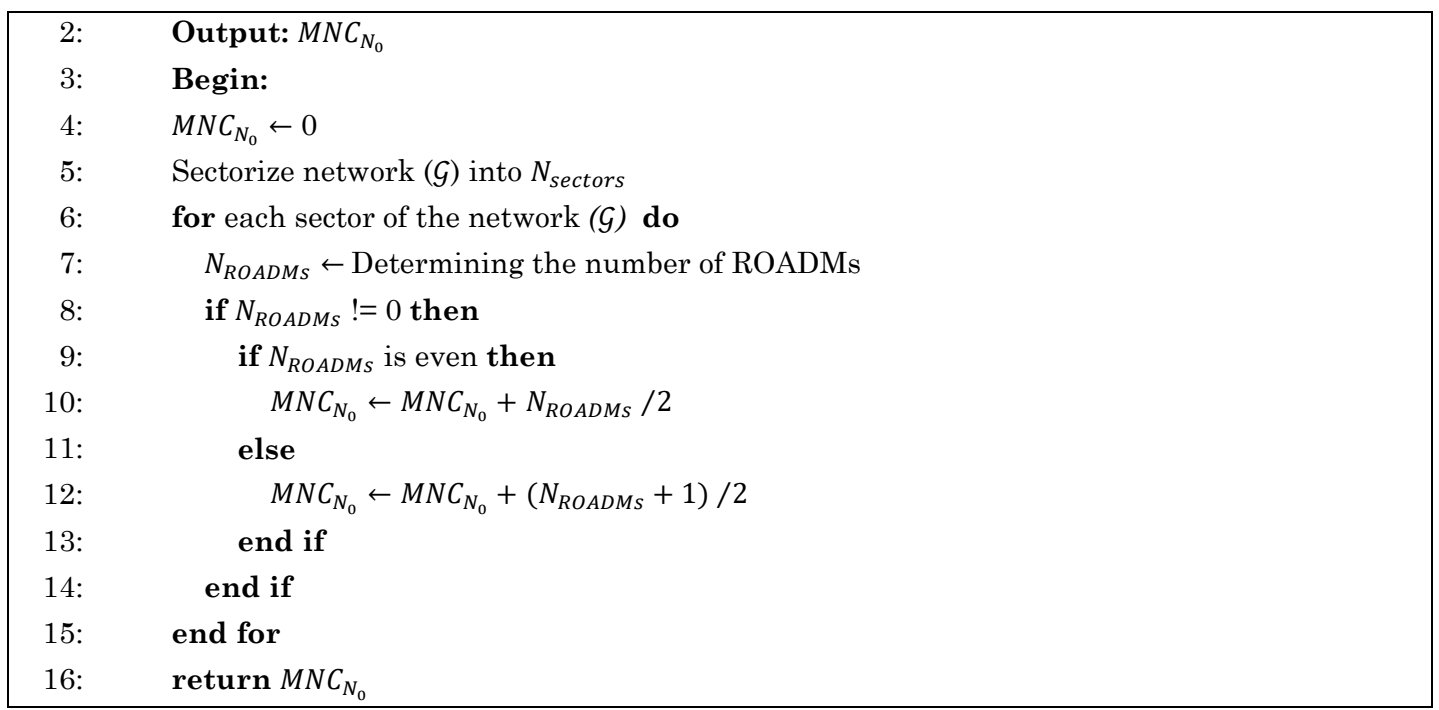

Fig. 6. Proposed pseudo-code of a heuristic that permits to determine the minimal number of channels required in a Metro-Access Network design in order to ensure that every connection between all possible pair of source-destination nodes can be established.

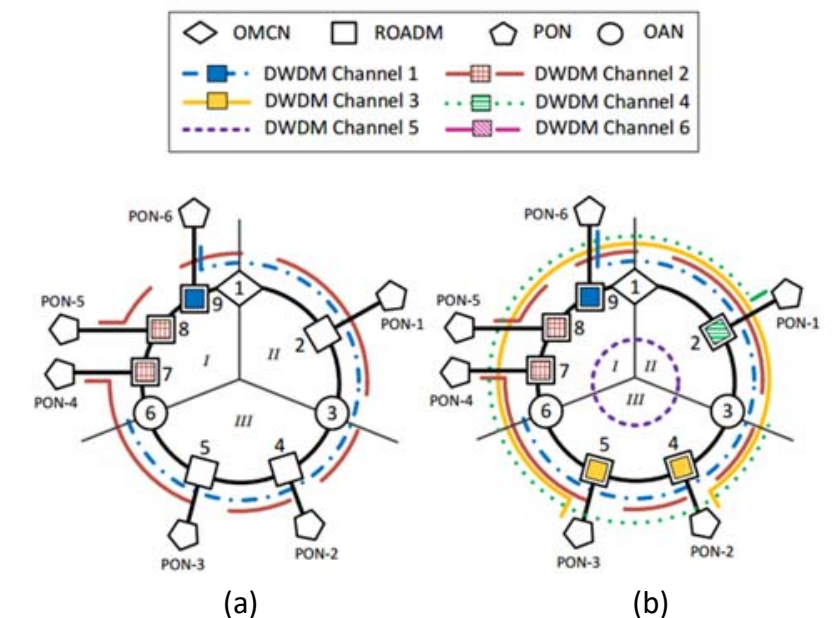

Fig. 7. (a) An example of sectioning of a network and (b) its initial design.

Once the minimum number of DWDM channels $\left(\mathrm{MNC}_{\mathrm{N}_{0}}\right)$ has been calculated to ensure that there are logical paths to establish any possible connection in the network, the required minimal network bandwidth $\left(\mathrm{BW}_{\mathrm{N}_{\mathrm{o}}}\right)$ can be calculated as $\mathrm{BW}_{\mathrm{N}_{0}}=\mathrm{MNC}_{\mathrm{N}_{0}} * \mathrm{BW}_{\mathrm{DWDM}-\mathrm{C}}$. Therefore, for a predefined value of the $\mathrm{BW}_{\mathrm{DWDM}-\mathrm{C}}$, if $\mathrm{BW}_{\mathrm{N}_{\mathrm{o}}}$ is higher than $\mathrm{BW}_{\mathrm{N}}$, more network bandwidth is required or smaller BW $\mathrm{DWDM}_{\mathrm{C}}$, finer DWDM granularity, has to be considered. In other cases, an initial design of the network and its ROADMs configuration can be obtained. For the example shown in Fig. 7(a), the initial design of the network is shown in Fig. 7(b). It is the simplest solution in the design of a Metro-Access Network based on DROADMs. This initial solution can be a proper solution as long as its $\mathrm{P}_{\mathrm{WC}}$ value is lower than the considered power budget $\left(\mathrm{PB}_{\mathrm{o}}\right)$; otherwise more sophisticated transmission technologies, providing a higher power budget, would be required.

Starting from the configuration of the D-ROADMs obtained in the previous problem, a second sub-problem involves obtaining a better configuration for the mismatched unpaired D- 
ROADMs of the network (e.g. D-ROADMs with IDs 2 and 9 shown in Fig. 7). A way to reach that purpose can be the sharing of the channel assigned to a mismatched ROADM of a sector with another mismatched ROADM of the other sector. That is, referring to the network example shown in Fig. 7(b), the DWDM channel 1 can be assigned to the ROADM 2 and the DWDM channel 4 to the ROADM 9, realizing a new design for those ROADMs as it can be seen in Fig. 8(a). To ensure that any connection between a node of PON-1 or PON-6 and a node of the ring can be established, the switches of ROADM's modules should have opposite configurations as it is shown in Fig. 8(a). This strategy increases the flexibility of ROADMs 2 and 9 but the DWDM channel 4 is underused because it is only considered for allocating traffic demands between node 1 and nodes pertaining to PONs 1 and 6 [see Fig. 8(a)]. To cope with that problem, we must decide if the unused channel is assigned for allocating traffic demands between nodes of the ring part of the network or for allocating traffic demand from/to PONs. As it is indicated in Fig. 8(b), it was decided to re-assign the underused channel 4 to PON 4, adding a new module to ROADM 7.

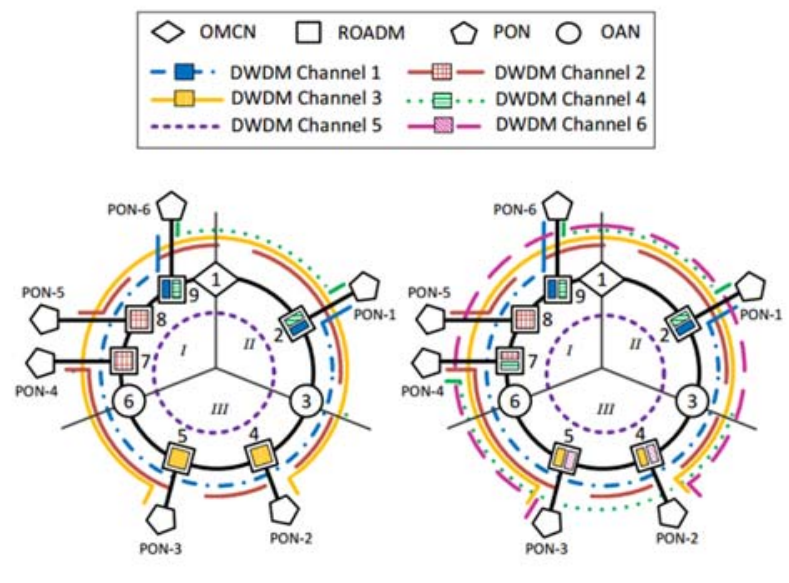

(a)

(b)

Fig. 8. (a) An example of how assigned channel to mismatched ROADMs of the network can be shared and (b) the final design of the considered network using the proposed heuristic II.

Once determined the best initial configuration of the network to ensure full connection between every pair of source-destination of the network and checking there are not underused channels, the third sub-problem involve assigning still available channels for increasing the TLC network so long the $\mathrm{P}_{\mathrm{WC}}$ of the current network design is lower than the considered $\mathrm{PB}_{\mathrm{o}}$. To decide in which part of the network the resources could be assigned, the RSA problem over the previous network design has to be solved in order to discover the most congested network's parts to add a new available DWDM channel. This is recursively done still exhausting the free DWDMs channels. Following with the proposed example, just one channel is available in this step, DWDM channel 6 [see Fig. 8(a)], so it is decided to assign it to ROADMs 4 and 5, as it can see in Fig 8(b). According to Fig. 5, the number of times that the dynamic RSA problem for all traffic demands has to be resolved to obtain the best network's design is also $2 *\left(M N C_{N}-M N C_{N_{0}}\right)+1$. 


\section{Scenario details and assumptions}

Fig. 9 shows two examples of ring-based Metro-Access Networks to analyse. The first one consists of one OMCN, two OANs and six ROADMs. The second one is based on one OMCN, five OANs and four ROADMs. In both cases, a Passive Optical Network (PON) with eight ONUs is considered to be connected to each ROADM.

For the sake of simplicity, we considered that the OMCN, OAN and ONU subsystems are able to add/remove specific channels to/from the network to which they are connected. In [7], we proposed a set of different cost-effective solutions for transmitters and receivers and their main attributes for those opto-electrical subsystems implementation. In addition, it is indicated that all those transmitters and receivers can be adapted to implement different $\mathrm{u}$ DWDM transmission techniques. We select the pair of transmitter and receiver which provides the highest power budget for all opto-electrical subsystems implementation. Following such premises, we consider that the OMCN and OAN, which are usually more expensive equipment, are implemented by Mach-Zehnder transmitters able to provide higher spectral efficient modulation formats as Nyquist-DPSK. On the other hand, ONU transmitters, much more cost sensitive, could be based on Distributed Feedback Lasers (DFB). The chosen receivers are based on a coherent receiver in which the detected signal is mixed with a local oscillator. As a reference, a DFB local oscillator has been considered, providing a sensitivity of $-52 \mathrm{dBm}[5,7]$. Therefore, the power budget $\left(\mathrm{PB}_{0}\right)$ is $52 \mathrm{~dB}$ for transmitters providing $0 \mathrm{dBm}$ output.

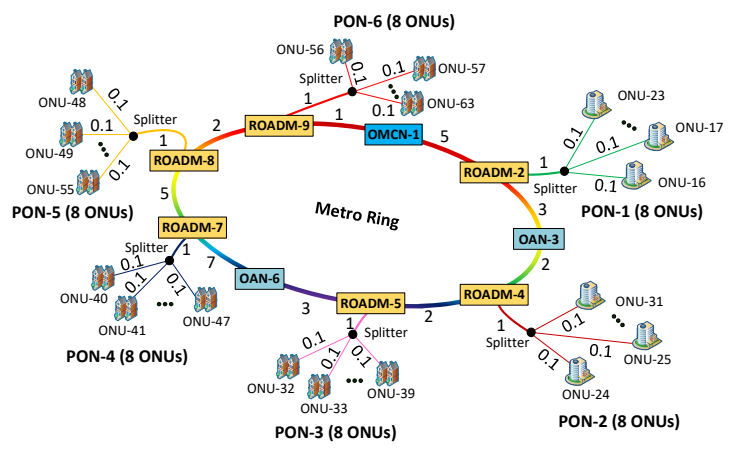

(a)

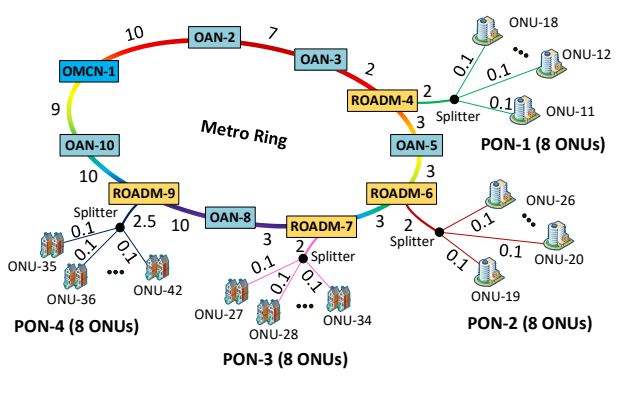

(b)

Fig. 9. Network examples considered (link distances are shown in km).

The power budget is considered to be consumed by the fiber attenuation $(0.25 \mathrm{~dB} / \mathrm{km}$ is assumed), the power splitter settled in each PON and the ROADMs' insertion loss [9]. Chromatic Dispersion (CD) effects due to the optical fiber are not considered in the study because it can be compensated using Digital Signal Processing (DSP) at the receiver [11].

A work band of $750 \mathrm{GHz}$ has been considered as the networks resource to share between all users. That work band is considered in order to reduce the computational cost. However, results would scale linearly for higher work bands. Two DWDM channel widths have been considered: $62.5 \mathrm{GHz}$ and $125 \mathrm{GHz}$; being $125 \mathrm{GHz}$ the bandwidth of the cost-effective DWDM ROADM proposed in [9] and $62.5 \mathrm{GHz}$ has been also considered for exploring a finer granularity. Each DWDM channel has been divided into frequency slots, being all of them of 12.5GHz [12].

The aforementioned networks can be loaded with dynamic traffic demands distributed uniformly between every pair of source-destination of the networks. Nevertheless, in that 
considered scenario, more than $90 \%$ of the traffic demands loaded in the network examples have their origin or destination in a node belonging to a PON, being that case unrealistic for a Metro-Access Network scenario as it is posed in Fig. 1. Therefore, the most suitable traffic demands distributions between every pair of networks nodes are considering that the probability for a demand presenting the origin or the destination in an ONU of a PON is 2.67 times lower than a demand that has origin and destination in the ring part of the network for the first network example (see Fig. 9(a)) and 3.33 times for the second network example (see Fig. 9(b)), respectively. These values are chosen in order to model the traffic generated by all ONUs of a PON as if they are a single node, reducing in this way the number of traffic demands which have the origin or the destination in a PON. In both considered network examples, the traffic demands arrival is a Poisson process with a given mean inter-arrival time (IAT) fixed to the unit and the durations (holding times, $\mathrm{HT}^{\mathrm{A}}=\mathrm{AIT}^{*} \rho$, where $\rho$ is the traffic load in erlang units) follow a negative exponential distribution. Two cases for the requested bitrate have been considered. In the first one all demands are of $10 \mathrm{Gbps}$ so each accepted demand takes up one $\mathrm{FS}$, and the second one where the requested bitrate is distributed among $\{10,20,40\} \mathrm{Gb} / \mathrm{s}$ with probabilities $\{0.5,0.25,0.25\}$. In all studied cases, the dynamic RSA problem, which is NP-hard, has been solved using a heuristical approach [10]. In particular, the two physically lowest loss paths (in $\mathrm{dB}$ ) have been pre-computed for every pair of source-destination of the network, and the spectrum resources, FSs, are allocated to the incoming demand following the first-fit policy. That heuristical approach delivers suboptimal solutions for large-moderate size problems [10].

\section{NUMERICAL RESULTS}

In this section, the results obtained for evaluating the proposed D-ROADM node architecture in single-fiber Metro-Access Network environments are analysed. While a more complex: double-fiber Metro + single-fiber Access could be considered, providing similar results, a homogeneous single-fiber Metro-Access Network scenario provides a simpler case to analyse. Moreover, full-duplex single-fiber Metro-Access has been demonstrated [13] and it is evolving towards higher spectral efficiency in (bits/s)/Hz.

First, we will evaluate the considered network scenarios, see Fig. 9, taking into account that the network bandwidth of $750 \mathrm{GHz}$ is divided into six DWDM channels of $125 \mathrm{GHz}$ and the bitrate of every traffic demand is of $10 \mathrm{Gbps}$. For this analysis, two cases will be discussed. A first one where the DWDM channels are assigned to the D-ROADMs of the network following the Heuristic I proposed in Section II.B, in which a same channel can be used for allocating traffic demands between nodes of the ring part of the network and between nodes belonging to a PON and a node of the ring. The second one consists on finding out the best configuration of each D-ROADM of the network in order to improve the TLC of the network following the proposed Heuristic II, introduced in the previous section. That is characterised by using different channels for allocating traffic demands between nodes of the ring and nodes of a PON, and for allocating in a different channel, traffic demands between nodes of the ring. Both cases will be compared to the reference, top performance and cost case [9], in which all the ROADMs are based on WSS and they can switch each frequency slot individually. A detailed cost comparison between D-ROADM and WSS-based ROADM has been made in [9]

Secondly, we will discuss the Blocking Bandwidth Probability (BBP) behaviour vs. TLC of the considered networks when the bandwidth of the DWDM channels is halved (from $125 \mathrm{GHz}$ to 
$62.5 \mathrm{GHz}$ ). Finally, the best configuration of the considered networks obtained after employing Heuristics I and II have been tested for a potential network evolution where bitrate of the demands can be $\{10,20,40\}$ Gbps with probabilities $\{0.5,0.25,0.25\}$, respectively. All results of this section have been obtained using a network simulator implemented in MATLAB ${ }^{\text {TM }}$ taking into account the insertion loss model of a D-ROADM proposed in [9] as well as considering $10^{5}$ offered traffic demands to the networks.

Figures 10(a) and 10(b) display the BBP as a function of the traffic that is loaded to the network when D-ROADM and WSS-based ROADMs are used for the case in which the bitrate of all traffic demands are of $10 \mathrm{Gbps}$ and the bandwidth of the DWDM channels are of $125 \mathrm{GHz}$. For the network based on D-ROADMs, the BBP is depicted for the proposed Heuristics I and II. From Figs. 10(a) and 10(b), we can notice that the proposed Heuristic II improves the TLC for the same BBP compared with the design derived from Heuristic I using the same spectral resources. For instance, the TLC obtained for Network I using Heuristic II when BBP $=5 \%$ is $53 \%$ higher than the TLC obtained when Heuristic $I$ is used. Similar BBP curve behaviour can be observed for Network II, in which the TLC when Heuristic II is used for a BBP $=5 \%$ is $63 \%$ higher than the TLC of the network configuration when Heuristic I is used. From Figs. 10(a) and 10(b), we can also notice that the performance penalty of the networks based on D-ROADM against the same networks using WSS-based ROADMs when the Heuristic II is used for $125 \mathrm{GHz}$ WDM channels is around $52 \%$, while for the Heuristic I the performance penalty is around $64 \%$.

Figures 10(a) and 10(b) also show the BBP curve when the considered DWDM channel bandwidth is of $62.5 \mathrm{GHz}$ and process of design of the D-ROADMs is based on Heuristic II. In this case, the performance penalty in the TLC of the considered networks is close to $35 \%$ (in average) regarding to the same networks using WSS-based ROADMs for a BBP value of 5\%, providing a significant improvement. This enhancement is due to a higher granularity and therefore the distribution of channels for each part of the network fits better to their bandwidth requirement.

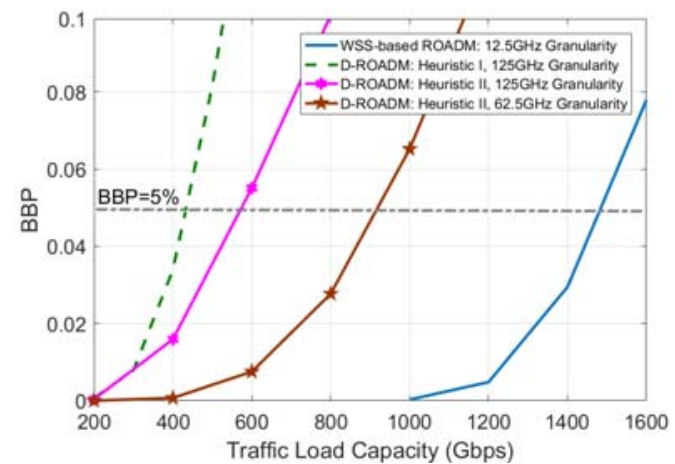

(a) Metro-Access Network I

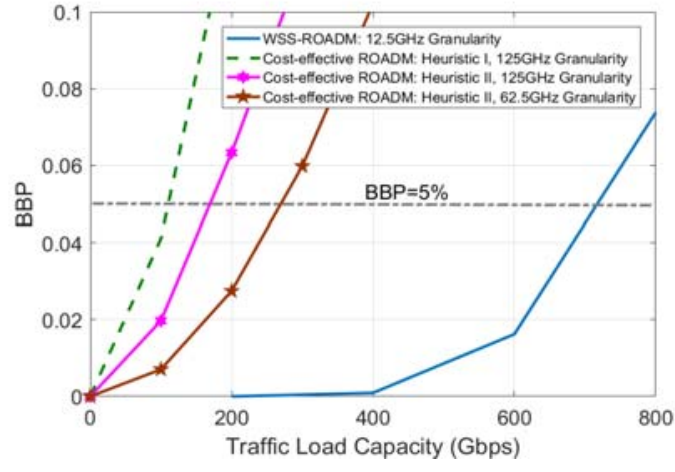

(a) Metro-Access Network I 


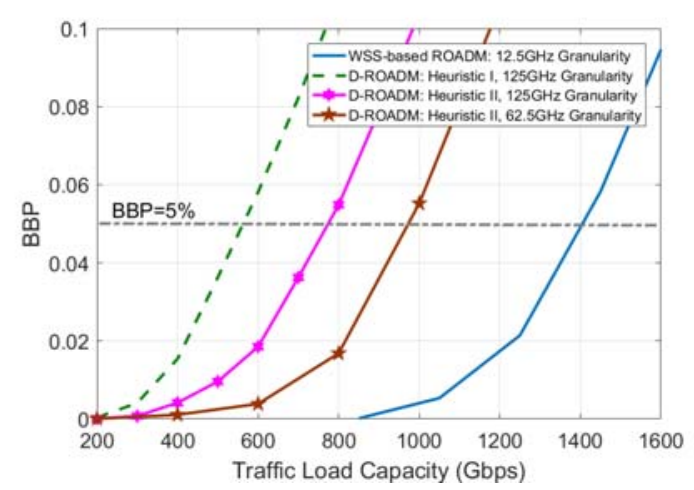

(b) Metro-Access Network II

Fig. 10. Blocking Bandwidth Probability (BBP) vs. Traffic Load Capacity (TLC) when bitrate of every traffic demand is of $10 \mathrm{Gbps}$ for considered MetroAccess Network I (a) and II (b).

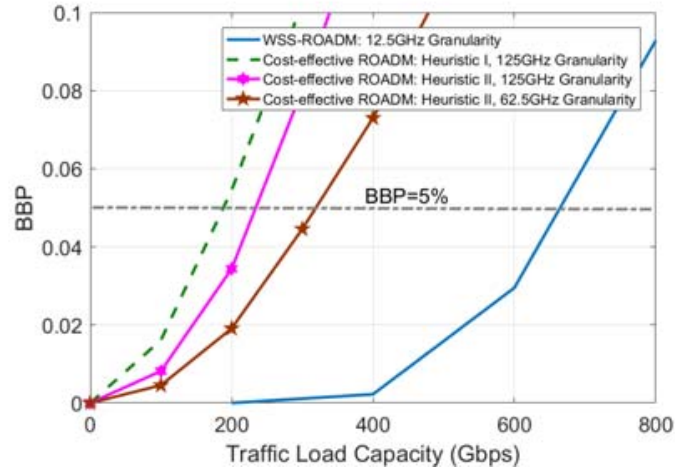

(b) Metro-Access Network II

Fig. 11. Blocking Bandwidth Probability (BBP) vs. Traffic Load Capacity (TLC) when bitrate of traffic demands can be $\{10,20,40\}$ Gbps for considered Metro-Access Network I (a) and II (b).

Table 2 shows the worst case path $\left(\mathrm{P}_{\mathrm{WC}}\right)$ of the considered networks (see Fig. 9) when they are constituted by WSS-based and D-ROADMs and designed by the two proposed heuristics, showing that is always well under the considered power budget of $52 \mathrm{~dB}$.

Figure 11 shows the BBP curves vs. TLC of the networks based on D-ROADMs after using both proposed heuristics as well as of the same networks using WSS-based ROADMs for the case in which the bitrate of the traffic demands can be $\{10,20,40\}$ Gbps with probabilities $\{0.5$, $0.25,0.25\}$, respectively. As we can notice from Figs. 11(a) and 11(b), using Heuristic II for the design of the D-ROADMs, the TLC is, in average, 1.43 times higher than Heuristic I for DWDM channel of $125 \mathrm{GHz}$; and 2.04 higher for DWDM channels of $62.5 \mathrm{GHz}$. The average penalty in the TLC of the D-ROADM-based networks using Heuristic II compared to the same networks using WSS-based ROADMs is of $72.2 \%$ and $59.2 \%$ for $125 \mathrm{GHz}$ and $62.5 \mathrm{GHz}$, respectively, as it can be deduce from Fig. 11. That performance loss is due to the fact that the D-ROADMs do not have the colorless feature.

\begin{tabular}{|c|c|c|c|c|c|c|c|c|c|c|}
\hline & \multicolumn{5}{|c|}{ Network I } & \multicolumn{5}{|c|}{ Network II } \\
\hline 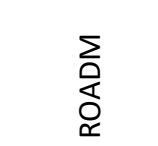 & 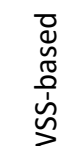 & \multicolumn{4}{|c|}{$\sum_{0}$} & 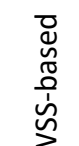 & \multicolumn{4}{|c|}{ 方 } \\
\hline $\begin{array}{c}\text { Granularity } \\
(\mathrm{GHz})\end{array}$ & 12.5 & \multicolumn{2}{|c|}{62.5} & \multicolumn{2}{|c|}{125} & 12.5 & \multicolumn{2}{|c|}{62.5} & \multicolumn{2}{|c|}{125} \\
\hline Heuristic & - & I & II & 1 & II & - & 1 & II & 1 & II \\
\hline $\mathrm{P}_{\mathrm{WC}}(\mathrm{dB})$ & 47 & 28 & 35 & 21 & 26 & 24 & 40 & 39 & 31 & 35 \\
\hline
\end{tabular}

Table 2. The worst case path $\left(P_{W C}\right)$ of the considered networks when WSS-based ROADMs and DWDM ROADMs are used for different granularities. For DWDM ROADMs $P_{W C}$ depends on the Heuristic used.

\section{CONCLUSION}

In this paper, we show the cost-effective D-ROADM performance in a metro-access ring network scenario demonstrating that a proper heuristic design can provide a significant improvement, doubling the performance using same network resources and just a $35 \%$ 
performance penalty for a D-ROADM with DWDM channels of $62.5 \mathrm{GHz}$ compared with a fully capable, $12.5 \mathrm{GHz}$ granularity WSS-based ROADM design. In particular, network's Traffic Load Capacity for a Blocking Bandwidth probability $=5 \%$, has been used as reference. In order to verify the validity of these heuristics, two different metro-access networks have been analyzed. In both cases, allocating traffic demands between nodes of the ring part of the network and nodes of a PON, and for allocating in a different channel, traffic demands between nodes of the ring significantly improves the TLC.

\section{ACKNOWLEDGEMENTS}

This work was supported in part ALLIANCE-B (TEC2017-90034-C2-2-R) co-funded by FEDER and MINECO FPI-BES-2015-074302.

\section{REFERENCES}

[1] M. Fiorani, et al., "Challenges for $5 G$ transport networks," in Proc. ANTS, New Delhi. India, pp. 1-6, Dec. 2014.

[2] H. Song, B. Kim, and B. Mukherjee, "Long-reach optical access networks: a survey of research challenges, demonstrations, and bandwidth assignment mechanisms," IEEE Comm. Surv. \& Tutor. 12(1), 112-123, 2010.

[3] J. A. Lazaro, C. Bock, V. Polo, R. I. Martinez, and J. Prat, "Remotely amplified combined ring-tree dense access network architecture using reflective RSOA-based ONU," J. Opt. Netw. 6, 801-807, 2007.

[4] H. Rohde, et al., "Coherent Ultra Dense WDM technology for Next Generation Optical Metro and access Networks," J. Lightw. Technol., vol. 32, no. 10, pp. 2041-2052, 2014.

[5] J.A. Altabas, David Izquierdo, J.A. Lazaro, A. Lerin, F. Sotelo, and I. Garces, "1Gbps fullduplex links for ultra-dense-WDM $6.25 \mathrm{GHz}$ frequency slots in optical metro-access networks," OPTICS EXPRESS, Vol. 24, Issue 1, pp. 555-565, 2016.

[6] Altabas, Jose A., et al. "1Gbps full-duplex $5 \mathrm{GHz}$ frequency slots uDWDM flexible Metro/Access Networks based on VCSEL-RSOA transceiver," OptoElectronics and Communications Conference (OECC) held jointly with 2016 International Conference on Photonics in Switching (PS), 2016 21st. IEEE, 2016.

[7] S. Sarmiento, R. Montero, J. A. Altabas, D. Izquierdo, F. Agraz,A. Pagès, ... \& Garces, I. "SDN-enabled flexible optical node designs and transceivers for sustainable metro-access networks convergence," in 2016 18th International Conference on Transparent Optical Networks (ICTON), IEEE, 2016.

[8] Aleksic, Slavisa. "Towards fifth-generation (5G) optical transport networks," in 2015 17th International Conference on Transparent Optical Networks (ICTON). IEEE, 2015.

[9] S. Sarmiento, J. A. Altabas, D. Izquierdo, I. Garces, S. Spadaro and J. A. Lazaro, "Costeffective DWDM ROADM design for flexible sustainable optical metro-access networks," in IEEE/OSA Journal of Optical Communications and Networking, vol. 9, no. 12, pp. 11161124, Dec. 2017.

[10]X. Wang, K. Kuang, S. Wang, S. Xu, H. Liu and G. N. Liu, "Dynamic routing and spectrum allocation in elastic optical networks with mixed line rates," in IEEE/OSA Journal of Optical Communications and Networking, vol. 6, no. 12, pp. 1115-1127, Dec. 2014. 
[11]Savory, Seb J., et al. "Electronic compensation of chromatic dispersion using a digital coherent receiver," Optics express 15.5, pp. 2120-2126, 2007.

[12] ITU-T Recommendation, G.694.1 (2012).

[13] Altabas, J. A., Izquierdo, D., Lazaro, J. A., Lerin, A., Sotelo, F., \& Garces, I. (2016). 1Gbps full-duplex links for ultra-dense-WDM $6.25 \mathrm{GHz}$ frequency slots in optical metro-access networks. Optics express, 24(1), 555-565. 